\title{
Analysis Mathematical Model of Radicalization S(Susceptible) E(Extremists) R(Recruiters) I (Immunity) with Optimal Control
}

\author{
Dauliyatu Achsina and Mardlijah
}

\begin{abstract}
Radicalization is a process when people come to adopt increasingly extreme political or religious ideologies, radicalization almost occurs in almost all countries in the world. Seeing a number of cases in recent times, radicalization has become a major concern for the world, especially in the field of national security. Radicalization has become one of the focuses in the national security sector because it leads to acts of extremism, violence and terrorism. The level of radicalization is high in each year and continues to increase so special supervision is needed to control it because it causes huge financial losses. Therefore a preventive effort is needed to overcome this. Efforts to prevent radical movements have been widely used, ranging from direct or indirect, in addition some things have also been done directly by the government. So far it has not been seen how effective these efforts are. Radicalization is formed because of the influence of extremists and the recruiters group. Many individuals are affected and enter the group because they are influenced by the people in the group who are within their scope. To overcome these problems, a control is needed as an effort to prevent radicalism. Prevention efforts are in the form of strict sanctions given to recruiters. Next to find out how the influence of controls on individual groups of recruiters is needed a tool to represent the tool is a model. The mathematical model that is suitable for representing the appropriate problems of radicalization is the Susceptible (S) , Extremists (E) Recruiters (R), Immunity (I) model.
\end{abstract}

Index Terms-Susceptible (S), Extremists (E), Recruiters (R), Immunity (I).

\section{INTRODUCTION}

$\mathbf{R}$ ADICALIZATION is a process in which people come to adopt increasingly extreme political or religious ideologies [1]. Radicalization almost occurs in almost all countries in the world. Seeing a number of cases in recent times radicalization has become a major concern for the world especially in the field of national security.

Radicalization has become one of the focuses in the national security sector because it leads to acts of extremism, violence and terrorism [2]. The level of radicalization is high in each year and continues to increase so special supervision is needed to control it because it causes huge financial losses as mentioned by [3]. Therefore, a preventive effort is needed to overcome this. Efforts to prevent radical movements have been widely used, ranging from direct or indirect, in addition some things have also been done directly by the government.

The authors are with the Department of Mathematics, Institut Teknologi Sepuluh Nopember, Kampus ITS Sukolilo-Surabaya 60111, Indonesia e-mail: mardlijah@matematika.its.ac.id

Manuscript received December 17, 2018; accepted July 18, 2021.
So far it has not been seen how effective these efforts are. Radicalization is formed because of the influence of extremists and the recruiters group. Many individuals are affected and enter the group because they are influenced by the people in the group who are within their scope. But for individuals who have become recruiters and given a sanction or control.

This phenomenon can be represented by mathematical models. The mathematical model will then be used to further the behavior of individuals in the population. Some researchers have reviewed the research by adopting the model from the basic mathematical model of epidemiology or commonly referred to as the model of disease spread.

The model of the spread of infectious diseases has been introduced by Hetchcote [4], the simple model describes the spread of disease by infected individuals to susceptible groups of individuals. Furthermore, the model has developed further by taking into account several factors, one of the factors is the incubation period or commonly referred to as the exposed period [5]. Choisy et al. [6] explain in the model that infected individuals can recover and not get infected again.

Broadly speaking, the behavior of the model of the spread of the disease can be interpreted in the eight social groups, namely radicalism. In his research, [1] explained that groups of vulnerable individuals could be exposed to radicalism because of direct contact with individuals. Furthermore, the study was further developed by [7] by paying attention to treatments in extremist groups and recruiters, but in this study, they still considered the possibility of treatment individuals returning to being infected.

Furthermore, this research will be combined with these two insights where recovered individuals cannot be re-infected. So that the model becomes a Susceptible (S) model, Extremists (E) Recruiters (R) and Immunity (I). The dynamics of individual movements in each group are represented in Figure 1. Panfilov et al. [8] states that the system is an autonomous system in which the variable $t$ is implied.

As for the assumptions used to limit the model, the assumptions are:

1) The susceptible group is a population where individuals in the group have not been affected by extremist ideology.

2) New individuals who enter the vulnerable population at a rate of $\Lambda$.

3) All populations in the susceptible group are equally likely to have the possibility of being influenced by extremists and becoming prospective recruiters. 


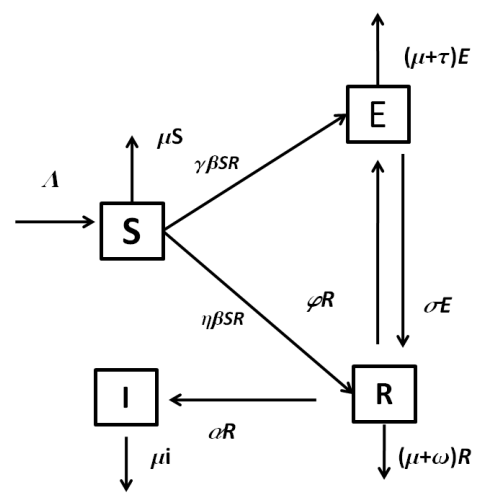

Fig. 1. Population Dynamics Spread of radicalism.

4) Extremists move to recruitment groups by $\sigma E$.

5) Recruiters can be extremists for $\varphi R$.

6) Individuals who already have immunity cannot be exposed to radicalism again.

The model describes the dynamics of population movements of each sub-population that occur because there is a contact between individuals from individuals extremsists to recruiters. So that individuals from the susceptible group can be influenced to become extremsist groups or recruiters groups.

Individuals born and entered into the population group are categorized as belonging to the susceptible population with the number of births is $\Lambda$.

Individuals in the naturally occurring susceptible group had a natural death rate of $\mu$ so the number of deaths in the susceptible group was $\mu S$.

The group of susceptible individuals can be reduced by the presence of susceptible individuals affected because of contact with individual extremists and recruiters as much as $\beta S R$, with $\gamma$ being the contact rate of the proportion of individuals who move from that group.

So that the dynamics of population displacement in the susceptible group are represented as follows.

$$
S^{\prime}=\Lambda-\mu S-\beta S R
$$

Individuals in the extremists group are individuals from the susceptible group that are affected by $\gamma \beta S R$. Individuals in the extremists group can be reduced because there is a natural death $(\mu)$, the termination of the group is $\tau E$, and the recruiters are $\sigma E$. Individuals in extremists who move into recruiters are $\varphi R$. So that the dynamics of population displacement in the susceptible group are represented as follows.

$$
E^{\prime}=\gamma \beta S R-(\mu+\tau+\sigma) E+\phi R
$$

Individuals in the recruiters group were individuals who came from groups of affected extremists of $\sigma E$. Individuals in the recruiters group can be reduced because there is a natural death $(\mu)$ so that the number of naturally occurring individuals in the group is $\mu R$. Individuals from the recruiters group can move to become extremists because of certain factors,
TABLE I

INFORMATION ABOUT PARAMETERS

\begin{tabular}{c||l}
\hline \multicolumn{1}{c||}{ Rate } & Detail \\
\hline \hline$\Lambda$ & $\begin{array}{l}\text { the birth rate which is assumed to enter the suscep- } \\
\text { tible (vulnerable) sub-population }\end{array}$ \\
\hline$\mu$ & $\begin{array}{l}\text { the birth rate which is assumed to enter the suscep- } \\
\text { tible (vulnerable) sub-population }\end{array}$ \\
\hline$\beta$ & $\begin{array}{l}\text { the rate of contact in vulnerable individuals into } \\
\text { groups of extremists and recruiters }\end{array}$ \\
\hline$\gamma$ & $\begin{array}{l}\text { the rate of contact in vulnerable individuals into } \\
\text { groups of extremists and recruiters }\end{array}$ \\
\hline$\omega$ & death rates due to other factors from recruiters \\
\hline$\tau$ & the elimination rate of extremists and recruiters \\
\hline$\varphi$ & the rate of movement of extremists to recruiters \\
\hline$\alpha$ & the rate of recovery from recruiters \\
\hline
\end{tabular}

individuals who move for $\varphi R$. So that the rate of migration of recruiters is represented as

$$
R^{\prime}=\eta \beta S R+\sigma E-(\mu+\omega+\varphi+\alpha) R
$$

And the immunity individuals, namely groups of individuals who have immunity so that the time given socialization is not affected by the effectiveness of socialization as big as and increase in the number of individuals from recruiters who experience recovery as much as $\alpha R$. In addition, individuals from the immunity group were also affected by natural deaths of that group of $\mu I$.

$$
I^{\prime}=\alpha R-\mu I
$$

So that the mathematical model is obtained in the form of ordinary differential equations as follows

$$
\begin{aligned}
S^{\prime} & =\Lambda-\mu S-\beta S R \\
E^{\prime} & =\gamma \beta S R-(\mu+\tau+\sigma) E+\phi R \\
R^{\prime} & =\eta \beta S R+\sigma E-(\mu+\omega+\varphi+\alpha) R \\
I^{\prime} & =\alpha R-\mu I
\end{aligned}
$$

Next refer to, Nastiti [9] to find out how the influence of control in detail from the model is used optimal control.

\section{Research Methodology}

There are several steps taken to construct a control system from the SERI model with the addition of controls. These steps are

1) Identify the phenomenon of radical spread events in an area or region in accordance with the SERI model.

2) Determine what parameters affect the model. Steps 1-2 are used to determine the radicalization model.

3) Determine the controls that are in accordance with the system.

4) Enter the control on the system. Step 3-4 is used to determine the effect of control on the model.

5) Apply the value of the parameter value to the control.

6) Representing the results of the control. Steps 5-6 are used to determine the effectiveness of the controls given. 


\section{RESUlts AND ANALYSis}

There are two equilibrium points. They are the endemic equilibrium point and the disease equilibrium point. In this section, we will discuss about disease-free equilibrium points. The solution which is an equilibrium point is obtained by evaluating the function of the system of differential equations. In summary, it can be written as follows

$$
\begin{aligned}
\Lambda-\mu S-\beta S R & =0 \\
\gamma \beta S R-(\mu+\tau+\sigma) E+\phi R & =0 \\
\eta \beta S R+\sigma E-(\mu+\omega+\varphi+\alpha) R & =0 \\
\alpha R-\mu I & =0
\end{aligned}
$$

\section{A. Disease-Free Equilibrium Point}

So that the value of the equilibrium point of the disease is obtained as follows

$$
S=\frac{\Lambda}{\mu}, \quad E=0, \quad I=0, \quad R=0
$$

\section{B. Endemic Equilibrium Point}

In addition to the disease-free equilibrium point the model has a second equilibrium point, which is an endemic equilibrium point. The endemic equilibrium point is

$$
\begin{aligned}
S & =\frac{\bar{\omega}+\tau(\psi+\omega+\mu)}{\beta(\gamma \sigma+\eta(\sigma+\tau+\mu))} \\
E & =-\frac{(\alpha \gamma+\eta \psi+\gamma(\psi+\omega+\mu)) \xi}{\Theta} \\
R & =\frac{\beta \Lambda(\gamma \sigma+\eta(\sigma+\tau+\mu)) \Gamma}{A+\tau(\psi+\omega+\mu)} \\
I & =\frac{\alpha(-\beta \Lambda(\gamma \sigma+\eta(\sigma+\tau+\mu)))+B}{D+\tau(\psi+\omega+\mu)}
\end{aligned}
$$

where

$$
\begin{aligned}
\bar{\omega}= & \sigma \omega+\sigma \mu+\psi \mu+\omega \mu+\mu^{2}+\alpha(\sigma+\tau+\mu) \\
\xi= & (-\beta \Lambda(\gamma \sigma+\eta(\sigma+\tau+\mu)))+\Psi \\
\Psi= & \mu\left(\sigma \omega+\sigma \mu+\psi \mu+\omega \mu+\mu^{2}+\alpha(\sigma+\tau+\mu)+\right. \\
& \tau(\psi+\omega+\mu)) \\
\Gamma=- & \mu\left(\sigma \omega+\sigma \mu+\psi \mu+\omega \mu+\mu^{2}+\alpha(\sigma+\tau+\mu)+\right. \\
& \tau(\psi+\omega+\mu)) \\
\Theta= & \beta(\gamma \sigma+\eta(\sigma+\tau+\mu))(\sigma \omega+\sigma \mu+\psi \mu+\omega \mu+ \\
& \left.\mu^{2}+\alpha(\sigma+\tau+\mu)+\tau(\psi+\omega+\mu)\right) \\
A= & \beta\left(\sigma \omega+\sigma \mu+\psi \mu+\omega \mu+\mu^{2}+\alpha(\sigma+\tau+\mu)\right) \\
B= & \mu\left(\sigma \omega+\sigma \mu+\psi \mu+\omega \mu+\mu^{2}+\alpha(\sigma+\tau+\mu)+\right. \\
& \tau(\psi+\omega+\mu)) \\
D= & \beta \mu\left(\sigma \omega+\sigma \mu+\psi \mu+\omega \mu+\mu^{2}+\alpha(\sigma+\tau+\mu)\right)
\end{aligned}
$$

\section{Stability of Disease-Free Equilibrium Point}

Next is the point to determine the stability of the two equilibrium points with the Jacobian matrix. From the point of disease-free equilibrium with value $S=\frac{\Lambda}{\mu}, E=0, I=0$, $R=0$ use the Jacobian Matrix then get it

$$
J=\left[\begin{array}{cccc}
-\mu & 0 & -\frac{\beta \Lambda}{\mu} & -v \\
0 & -\sigma-\tau-\mu & \psi+\frac{\beta \gamma \Lambda}{\mu} & 0 \\
0 & -\sigma & -\alpha-\psi-\omega+\frac{\beta \eta \Lambda}{\mu}-\mu & 0 \\
0 & 0 & \alpha & v-\mu
\end{array}\right]
$$

\section{Stability of Endemic Equilibrium Point}

Furthermore, for the endemic equilibrium point the stability type is determined using the Jacobian matrix as follows.

$$
J=\left[\begin{array}{llll}
a_{11} & a_{12} & a_{13} & a_{14} \\
a_{21} & a_{22} & a_{23} & a_{24} \\
a_{31} & a_{32} & a_{33} & a_{34} \\
a_{41} & a_{42} & a_{43} & a_{44}
\end{array}\right]
$$

where

$$
\begin{aligned}
a_{11}= & -\mu-\frac{\beta \Lambda(\gamma \sigma+\eta(\sigma+\tau+\mu))-v}{o} \\
a_{21}= & \frac{\gamma(\beta \Lambda(\gamma \sigma+\eta(\sigma+\tau+\mu))-y)}{\sigma \omega+\sigma \mu+\psi \mu+\omega \mu+\mu^{2}+\alpha(\sigma+\tau+\mu)+\tau(\psi+\omega+\mu)} \\
a_{31}= & \frac{\eta(\beta \Lambda(\gamma \sigma+\eta(\sigma+\tau+\mu))-w)}{n+\tau(\psi+\omega+\mu)} \\
a_{31}= & -\frac{l+\tau(\psi+\omega+\mu)}{\gamma \sigma+\eta(\sigma+\tau+\mu)} \\
a_{32}= & \psi+\frac{g}{\gamma \sigma+\eta(\sigma+\tau+\mu)}+p \\
a_{33}= & -\alpha-\psi-\omega-\mu \\
a_{22}= & -\sigma-\tau-\mu \\
a_{23}= & \sigma \\
a_{32}= & \alpha \\
a_{44}= & \mu \\
a_{41}= & 0, \quad a_{42}=0, \quad a_{14}=0 \\
a_{41}= & 0, \quad a_{42}=0, \quad a_{43}=0 \\
\rho= & \sigma+\tau+\mu \\
v= & \mu\left(\sigma \omega+\sigma \mu+\psi \mu+\omega \mu+\mu^{2}+\alpha(\sigma+\tau+\mu)+\right. \\
& \tau(\psi+\omega+\mu)) \\
o= & \sigma \omega+\sigma \mu+\psi \mu+\omega \mu+\mu^{2}+\alpha(\sigma+\tau+\mu)+ \\
& \tau(\psi+\omega+\mu) \\
y= & \mu\left(\sigma \omega+\sigma \mu+\psi \mu+\omega \mu+\mu^{2}+\alpha(\sigma+\tau+\mu)+\right. \\
& \tau(\psi+\omega+\mu)) \\
w= & \mu\left(\sigma \omega+\sigma \mu+\psi \mu+\omega \mu+\mu^{2}+\alpha(\sigma+\tau+\mu)+\right. \\
& \tau(\psi+\omega+\mu)) \\
n= & \sigma \omega+\sigma \mu+\psi \mu+\omega \mu+\mu^{2}+\alpha(\sigma+\tau+\mu) \\
l= & \sigma \omega+\sigma \mu+\psi \mu+\omega \mu+\mu^{2}+\alpha(\sigma+\tau+\mu) \\
g= & \gamma\left(\sigma \omega+\sigma \mu+\psi \mu+\omega \mu+\mu^{2}+\alpha(\sigma+\tau+\mu)+\right. \\
p= & \frac{\eta\left(\sigma \omega+\sigma \mu+\psi \mu+\omega \mu+\mu^{2}+\alpha(\rho)+\tau(\psi+\omega+\mu)\right)}{\gamma \sigma+\eta(\sigma+\tau+\mu)} \\
&
\end{aligned}
$$




\section{E. Optimal Control}

Furthermore, further analysis will be carried out on the model of spreading radicalism by using optimal control. The model without control is

$$
\begin{aligned}
S^{\prime} & =\Lambda-\mu S-\beta S R \\
E^{\prime} & =\gamma \beta S R-(\mu+\tau+\sigma) E+\phi R \\
R^{\prime} & =\eta \beta S R+\sigma E-(\mu+\omega+\varphi+\alpha) R \\
I^{\prime} & =\alpha R-\mu I
\end{aligned}
$$

Next is to maximize individual immunity by minimizing individuals whose recruiters with optimal control that is efficiently put on recruiters individuals, with the following controls $(u)$.

$$
\begin{aligned}
S^{\prime} & =\Lambda-\mu S-\beta S R \\
E^{\prime} & =\gamma \beta S R-(\mu+\tau+\sigma) E+\phi R \\
R^{\prime} & =\eta \beta S R+\sigma E-(\mu+\omega+\varphi+\alpha) R-u R \\
I^{\prime} & =\alpha R-\mu I+u R
\end{aligned}
$$

where $u$ is the control variable. Next we form the cost function as follows

$$
J=\int_{t_{0}}^{t_{f}} c_{1} R(t)+\frac{1}{2} c_{2} u^{2}(t) d t
$$

where $c_{1}$ is a weighting constant for effectiveness spread radicalism and $c_{2}$ is a weighting constant for cost. This function is a function to minimize with minimum Pontryagin.

With the limits $U, 0 \leq U \leq 1$ for control. Next we form the Hamiltonian function.

$$
\begin{aligned}
& H(x(t), u(t), \lambda(t), t)=V(x(t), u(t), t)+\rho(t) f(x(t), u(t), t) \\
& \quad c_{1} R(t)+\frac{1}{2} c_{2} u^{2}(t)+\lambda_{1}(\Lambda-\mu S-\beta S R)+ \\
& \quad \lambda_{2}(\gamma \beta S R-(\mu+\tau+\sigma) E+\varphi R)+ \\
& \quad \lambda_{3}(\eta \beta S R+\sigma E-(\mu+\omega+\varphi+\alpha)+ \\
& \quad \lambda_{4}(\alpha R-\mu I+u R)
\end{aligned}
$$

where $\lambda$ is a co state variable. For $i=1,2,3,4$ then to get the optimal conditions of $H(x(t), u(t), \rho(t), t)$ it must meet the stationary conditions of $H(x(t), u(t), \lambda(t), t)$. Here are the stationary conditions that must be met.

$$
\begin{aligned}
\frac{\partial H}{\partial u} & =0 \\
c_{2} u-\lambda_{3} R+\lambda_{4} R & =0 \\
c_{2} u & =\lambda_{3} R-\lambda_{4} R \\
u & =\frac{\lambda_{3} R+\lambda_{4} R}{c_{2}}
\end{aligned}
$$

Because the limit of the value of $u$ is $0 \leq u \leq 1$, there are several possibilities

$$
u=\left\{\begin{aligned}
\frac{\lambda_{3} R+\lambda_{4} R}{c_{2}}, & \text { if } 0<\frac{\lambda_{3} R+\lambda_{4} R}{c_{2}}<1 \\
0, & \text { if } \frac{\lambda_{3} R+\lambda_{4} R}{c_{2}} \leq 0 \\
1, & \text { if } \frac{\lambda_{3} R+\lambda_{4} R}{c_{2}} \geq 1
\end{aligned}\right.
$$

Of the several possibilities above, the optimal controller is

$$
u^{*}=\min \left\{1, \max \left\{0, \frac{\lambda_{3} R+\lambda_{4} R}{c_{2}}\right\}\right\}
$$

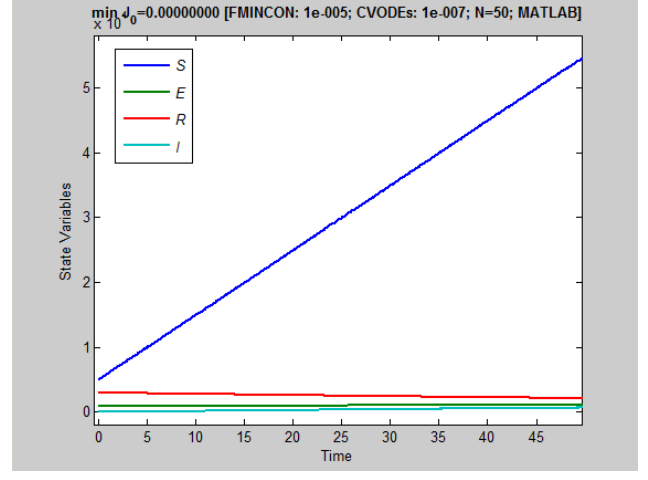

Fig. 2. Completion of the SERI Model before being influenced by control.

Then the equation state is

$$
x^{*}(t)=\frac{\partial H}{\partial x}
$$

Thus obtained

$$
\dot{x}=\left[\begin{array}{c}
\frac{\partial H}{\partial \lambda_{1}} \\
\frac{\partial H}{\partial \lambda_{2}} \\
\frac{\partial H}{\partial \lambda_{3}} \\
\frac{\partial H}{\partial \lambda_{4}}
\end{array}\right]=\left[\begin{array}{c}
\Lambda-\mu S-\beta S R \\
\gamma \beta S R-(\mu+\tau+\sigma) E+\phi R \\
\eta \beta S R+\sigma E-(\mu+\omega+\varphi+\alpha) R-u R \\
\alpha R-\mu I+u R
\end{array}\right]
$$

The equation of Co State

$$
\lambda^{*}=-\frac{\partial H}{\partial x}
$$

Thus obtained

$$
\begin{gathered}
\dot{x}=\left[\begin{array}{c}
\lambda_{1} \\
\lambda_{2} \\
\lambda_{3} \\
\lambda_{4}
\end{array}\right] \quad \begin{array}{c}
-\left(\lambda_{1}(\Lambda-\mu-\beta R)\right)+\lambda_{2}+h \\
-\left(\lambda_{2}(\gamma \beta S R-(\mu+\tau+\sigma)+\varphi R)+Z\right) \\
-\left(\begin{array}{c}
\left.\lambda_{1}(\Lambda-\mu S-\beta S-v I)+\lambda_{2}(\gamma \beta S-(\mu+\tau+\sigma) E+\varphi)\right)+M \\
-\left(\lambda_{1}(\Lambda-\mu S-\beta S R-v)+\lambda_{4}(v+\alpha R-\mu+u R)\right)
\end{array}\right]
\end{array}
\end{gathered}
$$

where

$$
\begin{gathered}
h=\lambda_{3}(\eta B R+\sigma E-(\mu+\omega+\varphi+\alpha) R-u R) \\
Z=\lambda_{3}(\eta \beta S R+\sigma-(\mu+\omega+\varphi+\alpha) R-u R) \\
M=\lambda_{3}(\eta \beta S+\sigma E-(\mu+\omega+\varphi+\alpha)-u)+ \\
\quad \lambda_{4}(\alpha R-\mu I+u)
\end{gathered}
$$

Furthermore, the model is simulated by using Matlab by entering parameter values taken from Clueskey and Santroprete [1] as follows $\Lambda=1000, \mu=0.00003427, \beta=$ $0.00000000056, S(0)=5000, I(0)=1000, E(0)=2000$, $R(0)=0$. As can be seen from Figure 2, the behavior of individual susceptible groups, extremist, recruiters and immunity before being given the influence of control.

Furthermore, in detail the effect of control in each compartment is explained in the figure 3.In the individual group susceptible there was no significant difference after being given control or before being given control. This is shown in the Figure 3 for the graph has not changed. 


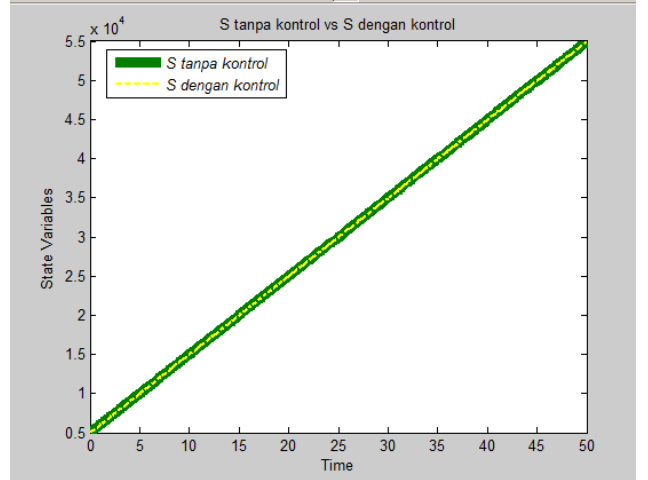

Fig. 3. Comparison of susceptible groups of individuals before and after influence of control.

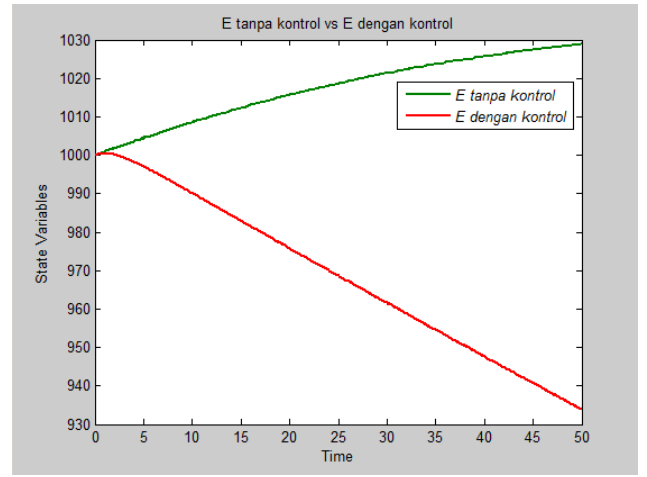

Fig. 4. Comparison of extrimist groups of individuals before and after influence of control.

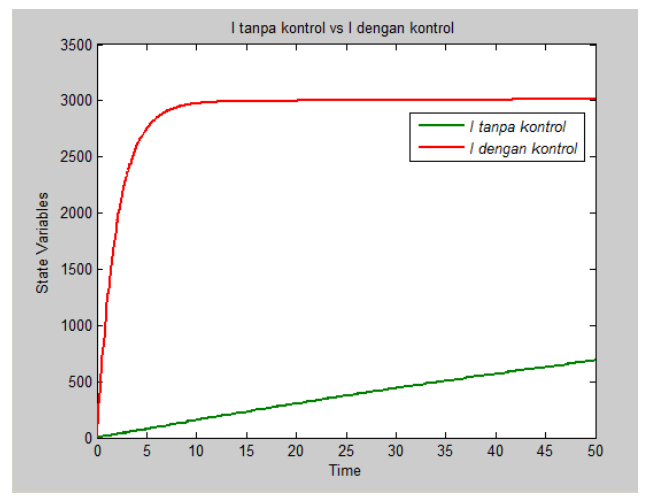

Fig. 5. Comparison of immunity groups of individuals before and after influence of control.

Furthermore, for estrimist individuals decline occurs at $t=$ 50 , with the influence of control it can be seen that the number 1000 starts to decrease dramatically from $t=0$ to $t=50$. The decline was due to the existence of estrimist individuals who became recruiters or died. It is shown at Figure 4.

Furthermore, control will be given to the model to determine the differences in recruiters individuals before being given control and as soon as given control. Then seen in Figure 6 There were significant differences between not yet given a control and after being given control. from the picture it can be seen that the behavior of recruiters individuals experience surging which initially 3000 at the same time has decreased

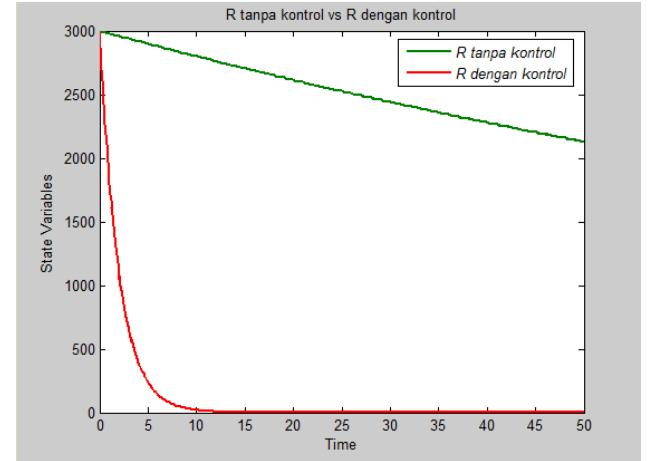

Fig. 6. Comparison of recruiters groups of individuals before and after influence of control.

TABLE II

SIMULATION

\begin{tabular}{c||c||c}
\hline$c_{1}$ & $c_{2}$ & Cost \\
\hline \hline 0.0000005 & 0.5 & 1503.55437745 \\
\hline 0.5 & 0.5 & 301.748888099 \\
\hline
\end{tabular}

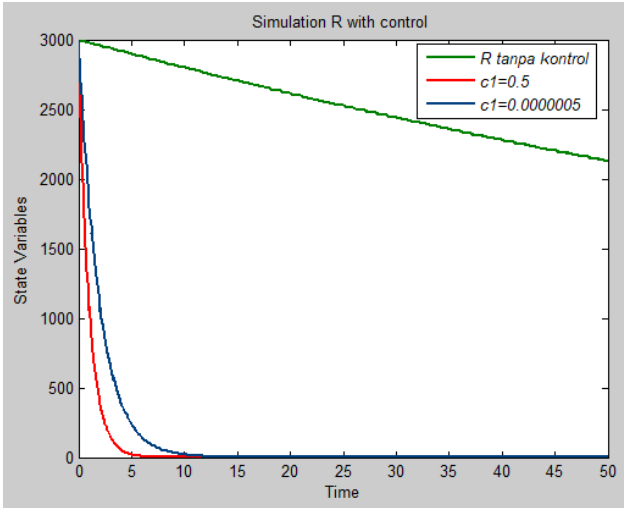

Fig. 7. Comparison of Recruiters groups of individuals before and after influence of control.

dramatically from $t=0$ to $t=50$. Then at $t=10$ the number of recruiters individuals begins to decline up to until $t=50$.

Then simulations will be carried out to find out more about how the controls affect the model. When given control, significant changes occurred in the recruiters individual group. Because control is given to recruiters so that control is very influential in reducing recruiters. Next will be simulated by taking different values with $c_{1}=0.0000005$ and $c_{2}=0.5$. The simulation results are represented in the Table II. To see a clearer reduction in the number of recruiters individuals are clarified in Figure 4.

Figure 7 explain overall about the difference in the decrease in the number of recruiters after the simulated. To see the details of the difference between the two retrieval values represented in the Figure 7. Seen by using the values $c_{1}=0.5$ and $c_{2}=0.5$ can reduce the number of recruiters individuals faster. for detailed comparison can be explained in Figure 8.

Furthermore, from Figure 9 is a graph of the control function $(u)$. It seems that the efforts made from the optimal start then over time began to decline due to the reduction in 


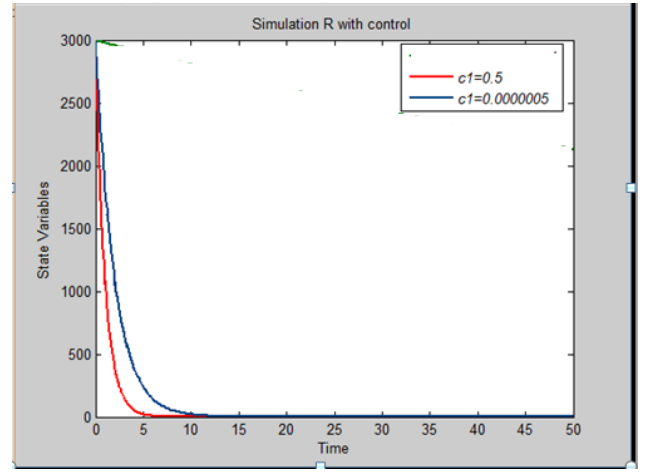

Fig. 8. Comparison of recruiters groups in different value of $c_{1}$.

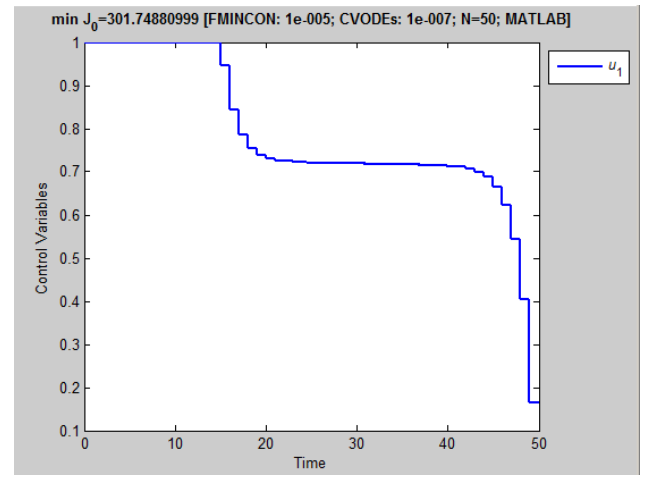

Fig. 9. Control Function with.

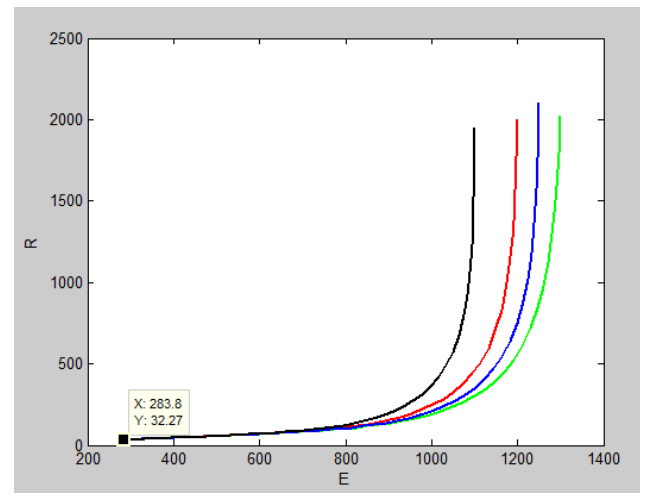

Fig. 10. Endemic Stability Phase Field.

TABLE III

Simulation initial VALUE

\begin{tabular}{c||c||c||c||c}
\hline$S(0)$ & $I(0)$ & $R(0)$ & $I(0)$ & Colour \\
\hline \hline 5000 & 1200 & 2000 & 0 & Black \\
\hline 5050 & 1300 & 2020 & 0 & Red \\
\hline 4900 & 1250 & 2100 & 0 & Blue \\
\hline 4950 & 1100 & 1950 & 0 & Green \\
\hline
\end{tabular}

recruiters individuals. Then to analyze how the behavior of the equilibrium point is stable or not by using the phase field with the initial condition shown at Table III. As can be seen on Figure 10.

From the simulation on Table II. Results by choosing three possible values from the weighting of the effectiveness of the number of recruiter and the costing weighting constants, the optimal effectiveness when $c_{1}=0.5, c_{2}=0.5$.

\section{CONCLUSION}

From the results of these studies the following conclusions can be drawn

1) There are two equilibrium points of this model

- Disease-free equilibrium

$$
S=\frac{\Lambda}{\mu}, \quad E=0, \quad I=0, \quad R=0
$$

- Endemic-free equilibrium

$$
\begin{aligned}
S & =\frac{\bar{\omega}+\tau(\psi+\omega+\mu)}{\beta(\gamma \sigma+\eta(\sigma+\tau+\mu))} \\
E & =-\frac{(\alpha \gamma+\eta \psi+\gamma(\psi+\omega+\mu)) \xi}{\Theta} \\
R & =\frac{\beta \Lambda(\gamma \sigma+\eta(\sigma+\tau+\mu)) \Gamma}{A+\tau(\psi+\omega+\mu)} \\
I & =\frac{\alpha(-\beta \Lambda(\gamma \sigma+\eta(\sigma+\tau+\mu)))+B}{D+\tau(\psi+\omega+\mu)}
\end{aligned}
$$

2) In the SERI model the control used to suppress recruiters is the number of individuals.

$$
u^{*}=\min \left\{1, \max \left\{0, \frac{\lambda_{3} k R+\lambda_{4} R}{c_{2}}\right\}\right\}
$$

3) In the simulation with parameter values, It can be seen that there are significant recruitment of recruiters when they are in control and there is a significant increase in individuals who have immunity and cannot be recruited again.

\section{REFERENCES}

[1] C. McCluskey and M. Santoprete, "A bare-bones mathematical model of radicalization," arXiv preprint arXiv:1711.03227, 2017.

[2] R. Rahimullah, S. Larmar, and M. Abdalla, "Understanding violent radicalization amongst muslims: A review of the literature," Journal of Psychology and Behavioral Science, vol. 1, no. 1, pp. 19-35, 2013.

[3] E. Mulcahy, S. Merrington, and P. Bell, "The radicalisation of prison inmates: A review of the literature on recruitment, religion and prisoner vulnerability," Journal of Human Security, vol. 9, no. 1, pp. 4-14, 2013.

[4] H. Hethcote, "The mathematics of infectious diseases," SIAM review, vol. 42, no. 4, pp. 599-653, 2000.

[5] O. Tessa, "Mathematical model for control of measles by vaccination," in Proceedings of Mali Symposium on Applied Sciences, vol. 2006, 2006, pp. 31-36.

[6] M. Choisy, J.-F. Guégan, and P. Rohani, "Dynamics of infectious diseases and pulse vaccination: teasing apart the embedded resonance effects," Physica D: Nonlinear Phenomena, vol. 223, no. 1, pp. 26-35, 2006.

[7] M. Santoprete and F. Xu, "Global stability in a mathematical model of de-radicalization," Physica A: Statistical Mechanics and its Applications, vol. 509, pp. 151-161, 2018.

[8] A. Panfilov, "Qualitative analysis of differential equations," arXiv preprint arXiv:1803.05291, 2018

[9] N. Nastitie and D. Arif, "Analysis and optimal control in the cancer treatment model with combining radio and anti-angiogenic therapy," IJCSAM (International Journal of Computing Science and Applied Mathematics), vol. 3, no. 2, pp. 55-60, 2017. 